\title{
HYDROGELS FROM N,N'-DIMETHYLACRYLAMIDE-co-2-ACRYLAMIDO-2-METHYL-1-PROPANESULFONIC ACID WITH SALT-, TEMPERATURE- AND pH-RESPONSIVENESS PROPERTIES
}

\author{
S. AMALIA POOLEY*I), BERNABÉ L. RIVAS ${ }^{(1)}$, AMITZA L. CÁRCAMO ${ }^{\prime)}$, GUADALUPE DEL C. PIZARRO \\ 1) Polymer Department, Faculty of Chemistry, University of Concepción, Casilla 160-C, Concepción, Chile. \\ ${ }^{2}$ Department of Chemistry, Technological Metropolitan University, J. P. \\ Alessandri 1242, Santiago, Chile \\ (Received: 17 December 2007 - Accepted: 10 March 2008)
}

\begin{abstract}
The synthesis of copolymer hydrogels of $N$, $N^{\prime}$-dimethylacrylamide (NNDMAAm) with 2-acrylamido-2-methyl-1-propanesulfonic acid (AMPS) were prepared by solution free radical polymerization at different feed mole ratios (mole ratios of $\mathrm{n}_{\text {NNDMAAm }} / \mathrm{n}_{\mathrm{AMPS}}=0.5 / 0.5 ; 0.33 / 0.66 ; 0.66 / 0.33 ; 0.25 / 0.75 ; 0.75 / 0.25$ ). In this reaction ammonium persulfate (APS) and $\mathrm{N}_{\mathrm{N}} \mathrm{N}^{\prime}$-methylene-bis-acrylamide (MBA) were used as initiator and cross-linking reagents respectively. These copolymers were insoluble in water and it were characterized by elemental analysis, FT-IR spectroscopy, thermogravimetric analysis (TGA), and scanning electron microscopy (SEM). Aqueous solutions of the copolymers showed lower critical solution temperature behavior (LCST). The phase transition temperatures of aqueous solutions of these copolymers increased with increasing of hydrophilic AMPS comonomer content in the copolymers.

The effects of reaction parameters, including the concentration of cross-linking reagent, the monomer concentration, $\mathrm{pH}$, temperature, salt solutions, and solvent polarity on the water absorption have been also studied. Under the experimental conditions, the copolymer poly(NNDMAAm-co-AMPS) with a feed mol ratio $1: 2$ presents the best swelling properties. The monomer reactivity ratios for poly(NNDMAAm-co-AMPS) are the following: $\mathrm{r}_{1}=0.217$ and $\mathrm{r}_{2}=2.20,\left(\mathrm{r}_{1} \mathrm{x} \mathrm{r}_{2}=0.48\right)$.
\end{abstract}

Keywords: Copolymerization; gels; swelling; water absorption.

\section{INTRODUCTION}

In recent years considerable research attention has been focused on the materials polymer, especially the hydrogels that can retain important fractions of water within their structure but do not dissolve into it. Hydrogel properties mainly depend on the degree of cross linked, the chemical composition of the polymeric chains, and the interaction of the network and surrounding liquids.

Hydrogels are three-dimensional polymer network characterized by a remarkable ability to absorb water. Hydrophilicity or high water retention of hydrogels is attributed to the presence of hydrophilic groups, such as carboxylic acids, amides, and alcohols ${ }^{1-4}$.

Polyelectrolyte hydrogels exhibit high swelling a the first order phase transition ( collapse) caused by a small changes in external parameters such as temperature, solvent composition, electric field, and etc 5,6 .

Copolymerization is an effective method of modify of physical properties of polymers and gels. Several authors ${ }^{7-10}$ have studied the temperature collapse in PIPAAm hydrogels, and they reported that while incorporation of a hydrophilic comonomer leads to a higher LCST, incorporation of a hydrophobic monomer decreases the critical temperature Tc. The reason for this sharp phase transition is a good balance between hydrophilic and hydrophobic interactions in the polymer ${ }^{11}$. It undergoes a temperature induced collapse from an extended coil into a globule structure, a transition revealed on the macroscopic scale by a sudden decrease of the degree of swelling of PNIPAAm gels 12-17. Raising the temperature of an aqueous PNIPAAm solution above the LCST causes a coil-to-globule transition, followed by a phase separation. This phase transition is accompanied by a release of water bound to the polymer chain, which is an endothermic process. In the literature some methods for the detection of the LCST have been also reported. For example, light scattering to detect the coilto-globule transition ${ }^{18}$, turbidimetric measurements to achieve phase transition ${ }^{19}$, or differential scanning calorimetry (DSC) to measure the transition heat ${ }^{20}$. The proposed application of these polymers is their use in the preparation of thermosensitive polymers (microsystems). As these devices are employed in real world environments, the copolymers will show temperatures far in excess of their LCSTs (typically ca. $30^{\circ} \mathrm{C}$ for PNIPAAm) ${ }^{11}$ in the course of their lifetimes. Thus, the thermal properties of the copolymers, especially the glass transition temperatures $(\mathrm{Tg})$ and the thermal decomposition were investigated.

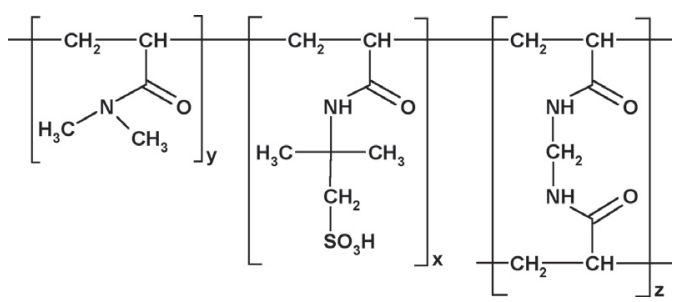

Poly(NNDMAAm-co-AMPS)

\section{EXPERIMENTAL PART}

\section{Materials}

$N, N^{\prime}$-dimethylacrylamide (NNDMAAm; Aldrich) was purified by distillation. All the other reagents were used as received without further purification.

Preparation of poly $\left[N, N^{\prime}\right.$-dimethylacrylamide-co-2-acrylamido-2-methyl1-propanesulfonic acid], poly(NNDMAAm-co-AMPS).

Cross-linked poly(NNDMAAm-co-AMPS) hydrogels, containing 25, 33, 50,66 , and $75 \mathrm{~mol} \%$ of NNDMAAm were prepared by solution free radical polymerization. NNDMAAm was dissolved in water, AMPS, MBA, and APS were added to the above NNDMAAm solution, the reaction solution was heated and polymerized for 24 hours at $70^{\circ} \mathrm{C}$ in a Teflon tube of $40 \mathrm{~mm}$ diameter. The product was cut into small discs $5 \mathrm{~mm} \times 20 \mathrm{~mm}$ and dried until constant weight. Finally, the dried product was characterized and the swelling properties were determined.

The feed mole ratios of NNDMAAm and AMPS are 1:1, 1:2, 1:3, 2:1, and $3: 1$. The total weight percentage of both monomers in the solution is $15 \%$. The weight percentage of the cross-linking reagent respect to the monomers is 2 ; $2.5 ; 3 ; 3.5 ; 4$ and 6 mol- $\%$. The weight percentage of the initiator respect to the monomers is always $1 \%$.

\section{Gel characterization}

The dried copolymers were ground to a suitably sized powder for FT-IR analysis. The FT-IR spectra of the copolymers were obtained with a Magna Nicolet IR-550 spectrophotometer.

\section{Measurements}

The elemental analyses were carried out using a 1108 FISON CE analyzer (Italy). FT-IR spectra were recorded on a Bruker model vector 22 Spectrophotometer (Bruker Optics Inc. USA).

\section{Thermal analysis}

The thermal analysis of the copolymers was determined by recording DSC under nitrogen atmosphere (flow rate $=150$ and $50 \mathrm{~cm}^{3} \mathrm{~min}^{-1}$, respectively). A sample size of 3-4 $\pm 0.1 \mathrm{mg}$ was used in each experiment. Thermal stability studies were performed using a Mettler Toledo Star System 822e (TG) (made in Spain) at a heating rate of $10^{\circ} \mathrm{C} / \mathrm{min}$. DSC measurements were carried to determine the glass transition temperature $\left(\mathrm{T}_{\mathrm{g}}\right)$ of the copolymer.

Thermogravimetry analysis of the copolymers was performed using TGA (Polymer Laboratories, STA-625 thermobalance). Measurements on $5 \mathrm{mg}$ of dry samples were carried out with a heating rate of $10{ }^{\circ} \mathrm{C} / \mathrm{min}$ from room temperature to $550^{\circ} \mathrm{C}$ under nitrogen atmosphere.

Copolymer morphology was examined by scanning electron microscopy (SEM) (Jeol, GSM-6380LV). 


\section{Swelling measurements}

The sample of poly(NNDMAAm-co-AMPS) $(0.5 \mathrm{~g})$ was immersed in $400 \mathrm{~mL}$ of distilled water for 12 hours until equilibrium was reached at room temperature. The weight of the swollen gel was measured after the excess surface solution was removed by filter paper. Then, the swollen gel was weighed. The absorbency was calculated using the following equation:

$\mathrm{Q}=\left(\mathrm{W}_{2}-\mathrm{W}_{1}\right) / \mathrm{W}_{1}$

Absorbency is expressed in grams of liquid retained in the gel per grams of dry copolymer. $\mathrm{W}_{2}$ and $\mathrm{W}_{1}$ are the weights of the swollen gel and the dry poly(NNDMAAm-co-AMPS), respectively.

\section{Effect of time, temperature, and $\mathrm{pH}$ on absorbency}

The methods were the same as used for the swelling measurements in twice distilled water, saline solution, and ethanol. The $\mathrm{pH}$ values of the solution were adjusted with dilute $\mathrm{HCl}$ or $\mathrm{NaOH}$.

\section{Water retention capacity}

The hydrogels were placed in twice-distilled water for one day. The swollen gels that reached equilibrium in water were drained in nylon bags for one day, then the gels and the bags were put into an oven and heated at constant temperature. To investigate the weight variation, the hydrogels were weighed at one day interval.

\section{RESULTS AND DISCUSSION}

Stimuli-responsive hydrogels change their volume and elasticity in response to a change in liquid phase properties, such as temperature, $\mathrm{pH}$, solvent composition, and ionic strength. Depending on the chemical composition of gels and liquid in experimental conditions, the change in the swelling behavior can occur either continuously or discontinuously.

The relationship between the gels' swelling behavior and the feed monomer ratios were studied at different temperatures and in different $\mathrm{pH}$ solutions. The transition temperature of the cross-linked gels changed according to the feed monomer ratio used in the copolymerization reaction. The $\mathrm{pH}$ value of the solution strongly affected the swelling ratio.

\section{Synthesis and characterization}

Copolymerization reactions were performed in water at different feed compositions, while maintaining constant the total amount of comonomers $(0.022 \mathrm{~mol})$. The yield for the all copolymer systems were higher that $90 \%$ (see table I).

Table I. Experimental conditions of the copolymerization reaction and yield.

\begin{tabular}{|c|c|c|c|c|c|c|}
\hline $\begin{array}{c}\text { Polymer } \\
\text { Sample } \\
\text { No }\end{array}$ & $\begin{array}{c}\text { Feed } \\
\text { monomer } \\
\text { ratio (in } \\
\text { mol) }\end{array}$ & $\begin{array}{c}\text { [NNDM } \\
\text { AAm }] \\
(\mathrm{mol})\end{array}$ & $\begin{array}{c}\text { [AMPS] } \\
(\mathrm{mol})\end{array}$ & $\begin{array}{c}{[\mathrm{MBA}]} \\
(\mathrm{mol})\end{array}$ & $\begin{array}{c}{[\mathrm{PSA}]} \\
(\mathrm{mol})\end{array}$ & $\begin{array}{c}\text { Yield } \\
(\%)\end{array}$ \\
\hline 1 & $(1: 1)$ & 0.0111 & 0.0111 & $8.87 \times 10^{-5}$ & $2.22 \times 10^{-5}$ & 86.8 \\
\hline 2 & $(1: 2)$ & 0.0074 & 0.0148 & $8.87 \times 10^{-5}$ & $2.22 \times 10^{-5}$ & 99.9 \\
\hline 3 & $(1: 3)$ & 0.0055 & 0.0166 & $8.87 \times 10^{-5}$ & $2.22 \times 10^{-5}$ & 96.9 \\
\hline 4 & $(2: 1)$ & 0.0148 & 0.0074 & $8.87 \times 10^{-5}$ & $2.22 \times 10^{-5}$ & 99.9 \\
\hline 5 & $(3: 1)$ & 0.0166 & 0.0055 & $8.87 \times 10^{-5}$ & $2.22 \times 10^{-5}$ & 98.0 \\
\hline
\end{tabular}

Hydrogels obtained in this study are transparent, smooth, and maintain their shape in the swollen state.

Figure 1 shows the FT-IR spectra of the five copolymers poly(NNDMAAmco-AMPS). The spectra show the typical absorption bands of the five comonomers and the cross-linking reagent. Among the most characteristic absorption bands are the following (in $\left.\mathrm{cm}^{-1}\right): 3450-3100(\mathrm{O}-\mathrm{H}$ stretching of acid group); 2940, 2810 (C-H stretching, aliphatic.); 1790,1640 (C=O group ester); 1643, 1550 (N-H deformation of amide group); 1219, 1034, 626 (stretching $\mathrm{SO}_{3}, \mathrm{~S}=\mathrm{O}$ and C-S from sulfonic group).

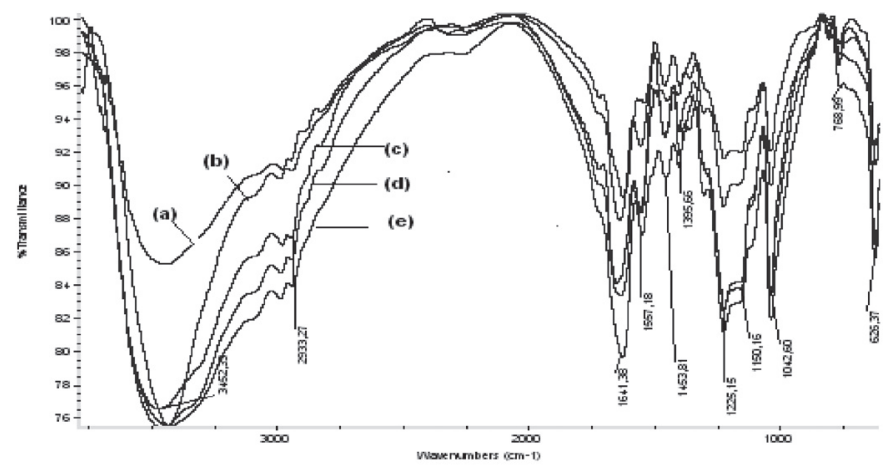

Figure 1. FTIR (KBr) spectra for poly(NNDMAAm-co-AMPS)1:1(a); 1:2(b); 2:1(c); and 3:1(e) 1:3(d);/4 mol-\% MBA.

The thermograms of all polymers show a typical sygmoidal shape. All the copolymers are degraded in one step and they are stable until $200^{\circ} \mathrm{C}$ with a weight-loss below $10 \%$ at $220^{\circ} \mathrm{C}$ (see Figure 2).

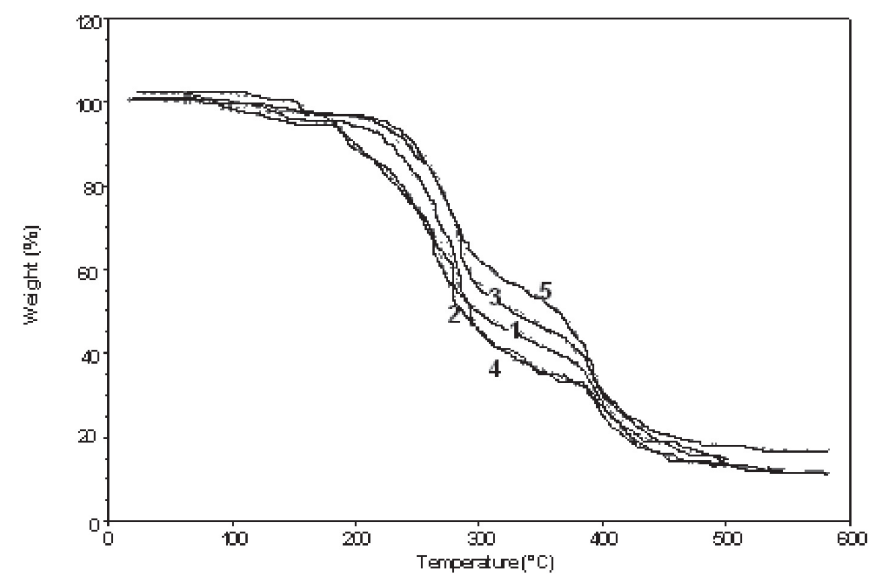

Figure 2. Thermal behavior of poly(NNDMAAm-co-AMPS) 1:1(1); $1: 2(2) ; 2: 1(3) ; 1: 3(4)$; and $3: 1(5) / 4$ mol- $\% \mathrm{MBA}$, heating rate: $10^{\circ} \mathrm{C} \mathrm{min}^{-1}$ under $\mathrm{N}_{2}$.

The typical scanning electron microscopy (SEM) of the poly(NNDMAAmco-AMPS) $1: 1 / 4 \%$ of MBA are shown in Figure 3, where the micrographs indicated a less-smooth morphology.

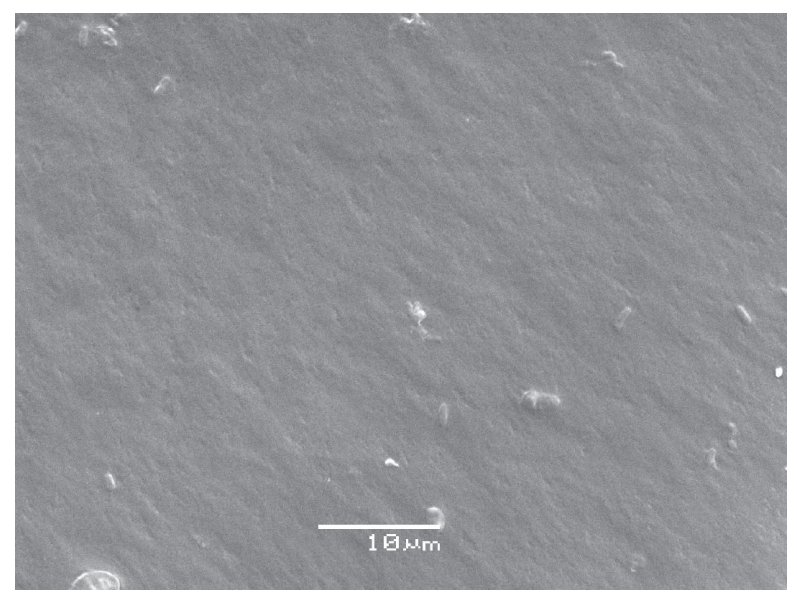

Poly(NNDMAAm-co-AMPS) 1:1/ 4\% MBA

Figure 3. SEM micrographs of poly(NNDMAAm-co-AMPS) 1:1 / 4 mol$\%$ MBA $(2000 x)$. 
Low Critical Solution Temperature (LCST)

It is found that aqueous solutions of these copolymers exhibited a lower critical solution temperature (LCST), depending on their chemical composition, followed by coacervate formation above the LCST. Furthermore, thermosensitive hydrogels could be prepared by the free radical copolymerization of NNDMAAm and AMPS in the presence of the cross-linking reagent MBA and initiator PSA. The poly(NNDMAAm-co-AMPS) showed a LCST around $40^{\circ} \mathrm{C}$, which was significantly higher than that poly(NIPAAm) ${ }^{11}$.

With an increase of NNDMAAm in the polymer, the phase transition temperature decreased due to the decrease of the hydrophilic balance, see Figure 4. The LSCT increased with an increasing the hydrophilic component of AMPS in the copolymer, see copolymers with initial feed composition 3:1; 1:1 and 1:3 respectively.

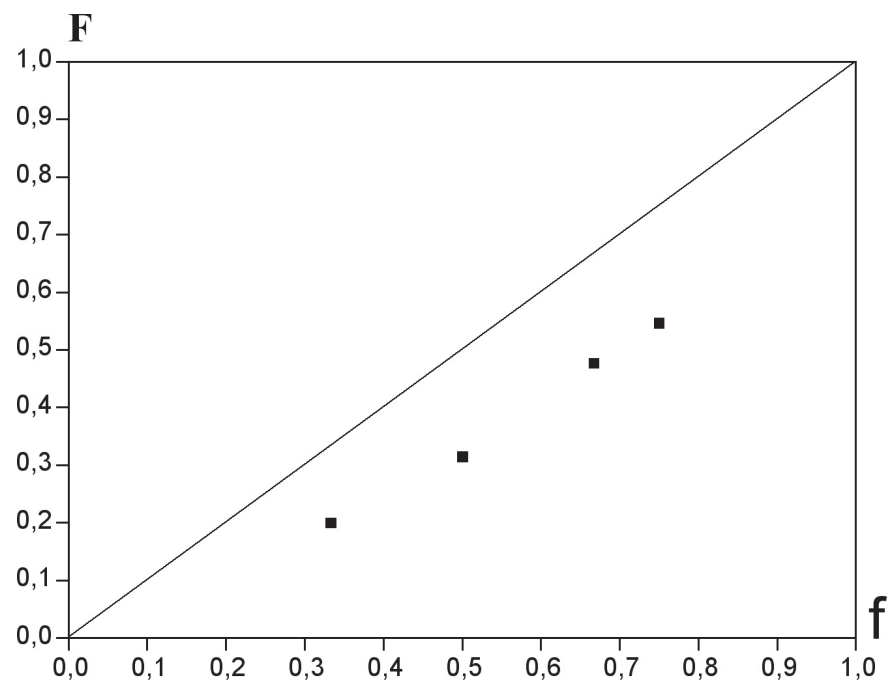

Figure 4. Relationship of M1 in the feed (M1) and in the copolymer (m1).

Monomer reactivity ratios, (MRR)

The monomer reactivity ratio values for the monomers NNDMAAm = (isopropylacrylamide) (PNIPAAm) ) and AMPS were determined from the monomer feed ratios and the copolymer composition obtained at relatively low conversions, according to Kelen Tüdös (K-T) method ${ }^{21}$. Copolymer composition were determined from elemental analysis data (see Table 2).

Table II. Elemental analysis, relationship S/C and copolymer composition results.

\begin{tabular}{|c|c|c|c|c|c|c|}
\hline \multirow{2}{*}{$\begin{array}{c}\text { Charge } \\
(\text { mol-\%) } \\
M_{1}: M_{2}\end{array}$} & \multirow{2}{*}{$\begin{array}{c}\text { Copolymer } \\
(\mathbf{m o l}-\%) \\
\left(\mathbf{m}_{1}\right)\end{array}$} & \multicolumn{4}{|c|}{$\begin{array}{c}\text { Elemental Analysis: Found (calculated) } \\
\text { value } \%\end{array}$} & \multirow[t]{2}{*}{$\begin{array}{l}\text { Relationship } \\
\text { S/C }\end{array}$} \\
\hline & & Sulfur & Carbon & Hydrogen & Nitrogen & \\
\hline $3: 1$ & 54.6 & $\begin{array}{c}9.616 \\
(\mathbf{9 . 8 2 5})\end{array}$ & $\begin{array}{c}46.851 \\
\mathbf{( 4 7 . 8 7 1 )}\end{array}$ & $\begin{array}{l}9.082 \\
(7.354)\end{array}$ & $\begin{array}{l}10.533 \\
(\mathbf{9 . 4 4 8 )}\end{array}$ & $\begin{array}{c}0.2052 \\
(\mathbf{0 . 2 0 5 2})\end{array}$ \\
\hline $2: 1$ & 47.7 & $\begin{array}{c}10.639 \\
(\mathbf{1 0 . 7 6 7 )}\end{array}$ & $\begin{array}{c}46.112 \\
\mathbf{( 4 6 . 6 5 3 )}\end{array}$ & $\begin{array}{l}9.318 \\
(\mathbf{7 . 1 8 2})\end{array}$ & $\begin{array}{c}9.973 \\
(\mathbf{8 . 9 9 9 )}\end{array}$ & $\begin{array}{c}0.2307 \\
(\mathbf{0 . 2 3 0 8})\end{array}$ \\
\hline $1: 1$ & 31.5 & $\begin{array}{c}12.485 \\
(\mathbf{1 2 . 6 8 6})\end{array}$ & $\begin{array}{c}43.466 \\
\mathbf{( 4 4 . 1 7 0 )}\end{array}$ & $\begin{array}{l}8.680 \\
(6.831)\end{array}$ & $\begin{array}{c}8.869 \\
\mathbf{( 8 . 0 8 5 )}\end{array}$ & $\begin{array}{c}0.2872 \\
(\mathbf{0 . 2 8 7 2})\end{array}$ \\
\hline $1: 2$ & 19.9 & $\begin{array}{c}13.067 \\
(\mathbf{1 3 . 8 2 1})\end{array}$ & $\begin{array}{c}40.372 \\
\mathbf{( 4 2 . 7 0 2 )}\end{array}$ & $\begin{array}{l}8.386 \\
(\mathbf{6 . 6 2 4})\end{array}$ & $\begin{array}{c}7.919 \\
(7.544)\end{array}$ & $\begin{array}{c}0.3237 \\
\mathbf{( 0 . 3 2 3 7 )}\end{array}$ \\
\hline $1: 3$ & 3.77 & $\begin{array}{c}13.447 \\
(\mathbf{1 5 . 1 8 5 )}\end{array}$ & $\begin{array}{c}36.251 \\
\mathbf{( 4 0 . 9 3 8 )}\end{array}$ & $\begin{array}{l}7.720 \\
(6.374)\end{array}$ & $\begin{array}{l}7.151 \\
(6.894)\end{array}$ & $\begin{array}{c}0.3710 \\
(\mathbf{0 . 3 7 0 9 )}\end{array}$ \\
\hline
\end{tabular}

M1: Initial composition of monomer 1 (NNDMAAm)

m1: copolymer composition of monomer 1 (NNDMAAm)

To determine the monomer reactivity ratios, a plot of monomer feed composition $\left(\mathrm{M}_{1}\right)$ vs monomer composition in the copolymer ( $\left.\mathrm{m}_{1}\right)$ (mol \%) for DMAEA is shown in Figure 5 for poly(NNDMAAm-co-AMPS). 


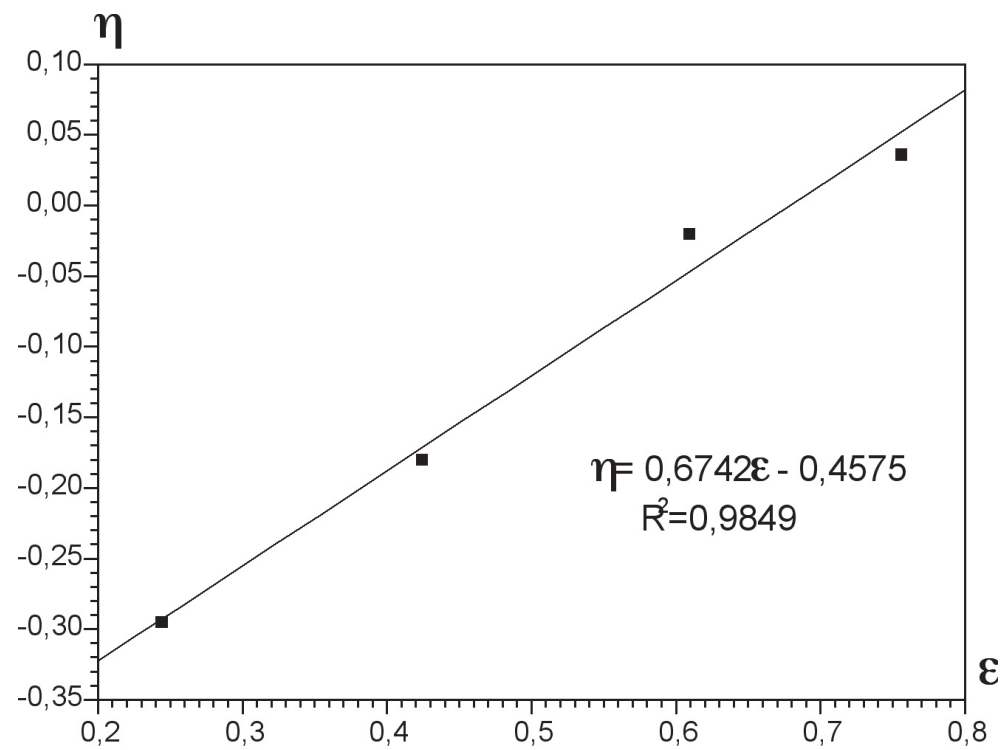

Figure 5. $\eta$ vs $\xi$ representation of the copolymerization parameters of poly(NNDMAAm-co-AMPS) by K-T method.

The Kelen Tüdös equation (1) is symmetrically transformed into

$$
G=r_{1} \times F-r_{2}
$$

by introducing the new parameters $\xi$, $\eta$, and $\alpha=\left(F_{\text {min }} \times F_{\text {max }}\right)^{0.5}$, where $\mathrm{F}_{\text {min }}$ and $\mathrm{F}_{\text {max }}$ correspond to the smallest and largest fraction in the copolymer (F) that are calculated, respectively (see Table 3).

Table III. Reactivity ratios of NNDMAAm and AMPS in poly(NNDMAAm -co-AMPS) by Kelen Tüdös for high conversion method, $\mu=2.0905, \alpha=$ 4.8024

\begin{tabular}{|c|c|c|c|c|c|c|c|c|c|c|c|}
\hline $\mathrm{M}_{1}^{\mathrm{a})}$ & $\mathrm{m}_{1}^{\mathrm{b})}$ & $\mathrm{wt}^{\mathrm{c})}$ & $\mathrm{Xo}$ & $\mathrm{y}$ & $\delta_{1}$ & $\delta_{2}$ & $\mathrm{Z}$ & $\mathrm{F}$ & $\mathrm{G}$ & $\xi$ & $\eta$ \\
& & & & & & & & & & \\
75.0 & 54.6 & 42.7 & 3.000 & 1.201 & 0.264 & 0.660 & 0.284 & 14.85 & 0.707 & \\
66.7 & 47.7 & 44.1 & 2.000 & 0.913 & 0.274 & 0.601 & 0.349 & 7.49 & -0.249 & 0.7550 .609 & -0.036 \\
50.0 & 31.5 & 46.7 & 1.000 & 0.459 & 0.260 & 0.566 & 0.360 & 3.538 & -1.503 & 0.424 & -0.180 \\
33.3 & 20.0 & 50.0 & 0.500 & 0.249 & 0.276 & 0.553 & 0.401 & 1.553 & -1.553 & $0.244 *$ & -0.295 \\
25.0 & 3.8 & 50.9 & 0.333 & 0.039 & 0.068 & 0.580 & 0.081 & 5.913 & 5.913 & & $*$ \\
& & & & & & & & & & & \\
\end{tabular}

a) Monomer composition in feed mol- $\%$; b) Copolymer composition of $\mathrm{M}_{1}$ in mol- $\%$

b) Conversion* values out side range

The transformed variables are defined as :

$\eta=\mathrm{G} /\left(\mathrm{r}_{1}+\mathrm{r}_{2} / \alpha\right) \quad \xi=\mathrm{F} /(\alpha+\mathrm{F})$

The $r_{1}$ and $r_{2}$ values were also determined according to Equation (2):

$\eta=\left(r_{1}+r_{2} / \alpha\right) \xi$

where $\eta$ and $\xi$ are mathematical functions of the monomer molar ratios in the feed and in the copolymer respectively, $\alpha$ is an arbitrary denominator with any positive value, which produces a more homogeneous distribution of data along $\eta-\xi$ axes.

The parameters $\xi, \eta$ and $\alpha$ have been previously defined: $\mathrm{G}=\left(\mathrm{m}_{1} / \mathrm{m}_{2}-1\right) / \mathrm{z}$ and $\mathrm{F}=\left(\mathrm{m}_{1} / \mathrm{m}_{2}\right) / \mathrm{z}^{2} ; \mathrm{z}=\log \left(1-\delta_{1}\right) / \log \left(1-\delta_{2}\right) ; \delta_{1}=\delta_{2} \mathrm{y} / \mathrm{X}_{0} ; \delta_{2}=\mathrm{wt} \%(\mu+\mathrm{X}) /(\mu$ $+\mathrm{y}) / 100 ; \mu=\mu_{2} / \mu_{1} ; \mathrm{y}=\mathrm{m}_{1} / \mathrm{m}_{2} ; \mathrm{X}_{\mathrm{o}}=\mathrm{M}_{1} / \mathrm{M}_{2} ; \mathrm{wt} \%=$ conversion; $\mu_{1}$ and $\mu_{2}$ are the molecular weights of monomers 1 and 2 respectively; $\mathrm{M}_{1}$ and $\mathrm{M}_{2}=$ initial composition of monomers in mol, $\mathrm{M}_{1}=$ poly(N-isopropylacrylamide) (PNIPAAm) (NNDMAAm) and $\mathrm{M}_{2}=$ AMPS ; $\mathrm{m}_{1}$ and $\mathrm{m}_{2}=$ correspond to the monomer composition in the copolymer for each monomer. The variable $\xi$ can take any possible value in the 0 to 1 interval. A plot of $\eta v s \xi$ gives a straight line, which on extrapolation to $\xi=0$ and $\xi=1$ gives $-r_{2} / \alpha$ and $r_{1}$, respectively (see Figure 6). 


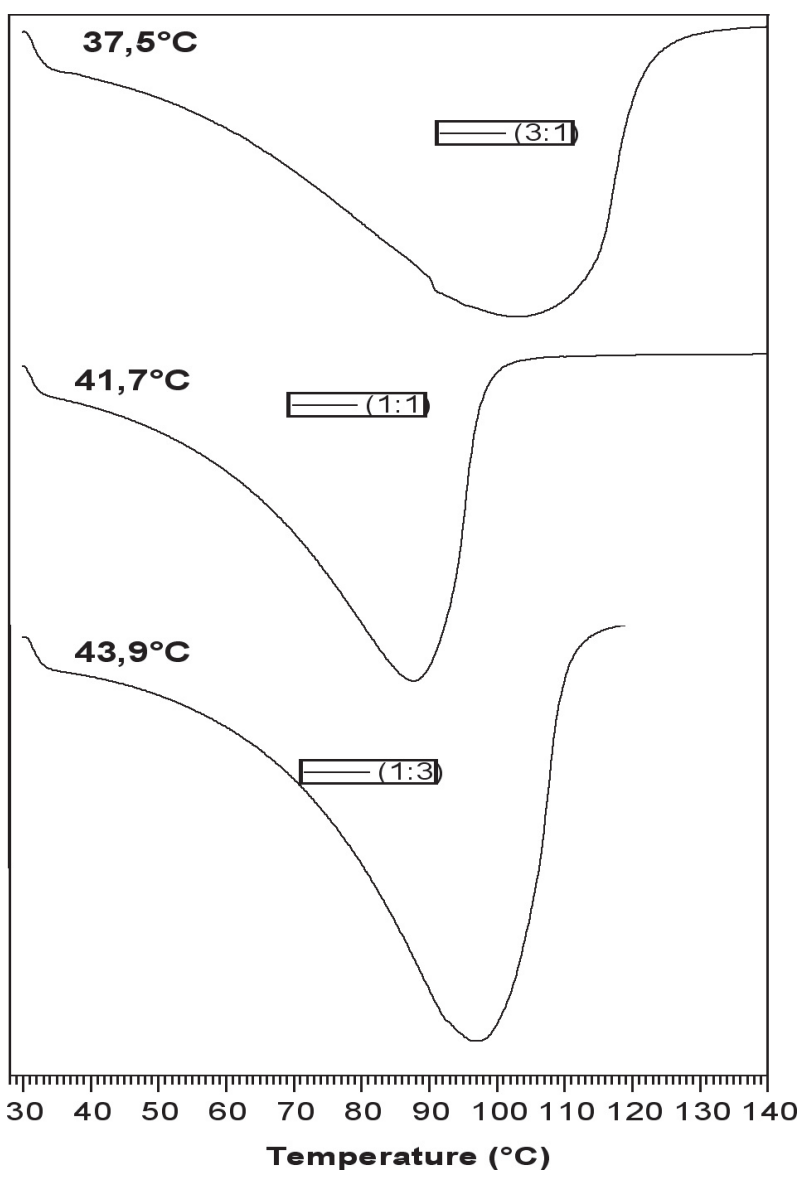

Figure 6. DSC thermograms of poly(NNDMAAm-co-AMPS) show a LCST around $40^{\circ} \mathrm{C}$. Feed monomer composition $3: 1,1: 1$, and 1:3 respectively. Heating rate: $10^{\circ} \mathrm{C} / \mathrm{min}$.

According to these values, the monomer reactivity ratios for poly(NNDMAAm-co-AMPS) are the following: $\mathrm{r}_{1}=0.217$ and $\mathrm{r}_{2}=2.20,\left(\mathrm{r}_{1} \mathrm{X}\right.$ $\mathrm{r}_{2}=0.48$ );

In general, these results suggest for poly(NNDMAAm-co-AMPS) system, that the chain growth reactions proceeds predominantly by the addition of NNDMAAm monomer to the $\sim$ R-AMPS ${ }^{*}$ macroradical, which AMPS possess a higher tendency of incorporation to the chain producing also random copolymers.

\section{Absorbency}

The absorbency of hydrogels in bidistilled water for five feed mol ratios is shown in Figure 7. As can be observed, poly(NNDMAAm-co-AMPS) 1:2 and 1:3 shown the highest $\mathrm{Q}$ values. This result is due to the gradual diffusion of water molecules into the network of the hydrogel and the complete filling or occupation of the pre-existing or dynamically formed spaces in the polymer chains.

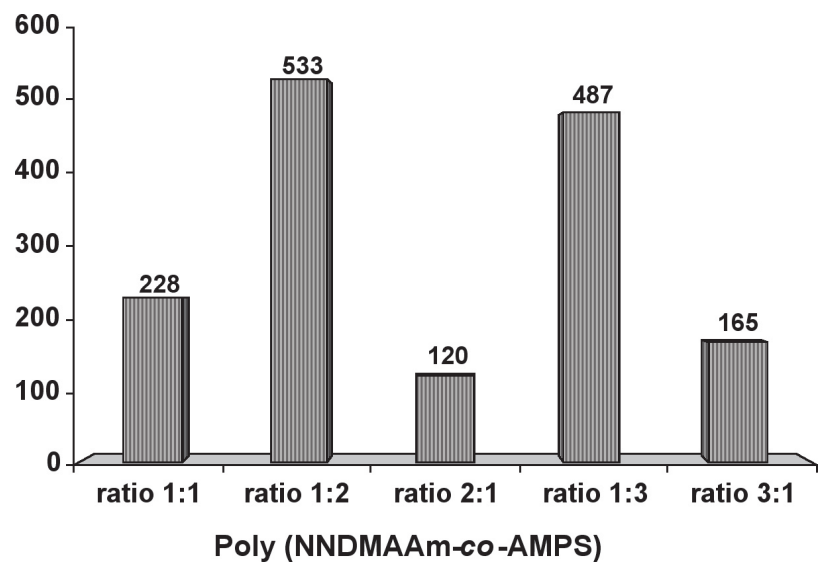

Figure 7. Absorption (Q) of poly(NNDMAAm-co-AMPS) $1: 1 ; 1: 2 ; 1: 3$; $2: 1 ; 3: 1 / 4$ mol- \% MBA bidistilled water at room temperature.

The absorbency of poly(NNDMAAm-co-AMPS) 1:2/2\% MBA is higher than that the other five copolymers in bidistilled water as well as ethanol at room temperature. It is shown in Figure 8. This result is due to the smaller amount of cross-linking incorporated into the backbone. This increase the space for the water molecules.

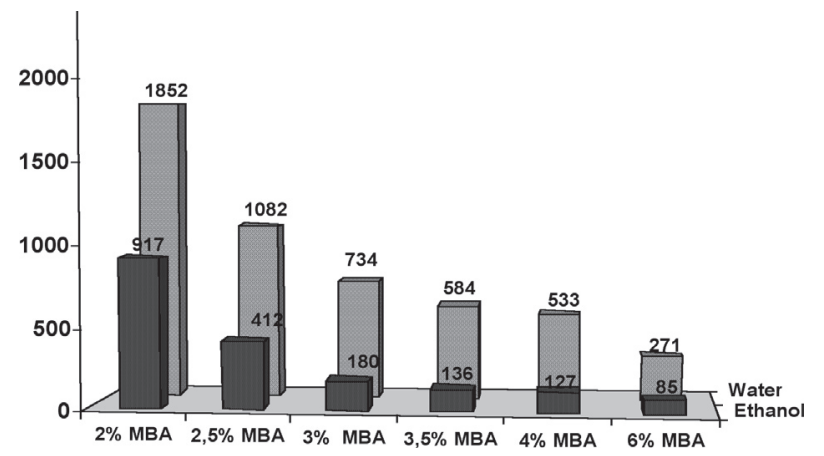

Figure 8. Absorption (Q) of poly(NNDMAAm-co-AMPS) 1:2 / 6; $4 ; 3.5 ; 3 ; 2.5$; and 2 mol- $\%$ MBA in bidistilled water and ethanol at room temperature

\section{Effect of pH on absorbency}

To investigate the influence of $\mathrm{pH}$ on the degree of gel swelling, the $\mathrm{pH}$ was adjusted with $1 \mathrm{M} \mathrm{HCl}$ or $1 \mathrm{M} \mathrm{NaOH}$ from $\mathrm{pH} 1$ to $\mathrm{pH}$ 9. Figure 9 shows the effects of the solutions' $\mathrm{pH}$ values on the swelling behavior for all the studied copolymers.

The absorption curve shows an optimal value at $\mathrm{pH} 5$ when the feed monomer ratio is 1:2 with 2.5 mol- $\%$ MBA. The absorbency increase from $\mathrm{pH}$ 1 to $\mathrm{pH} 5$ for all copolymers at $\mathrm{pH}$ more high the absorbency decrease abruptly for copolymers cross-linked with 2 mol- $\%$; 2.5 mol- $\%$; and 3 mol- $\%$ MBA.

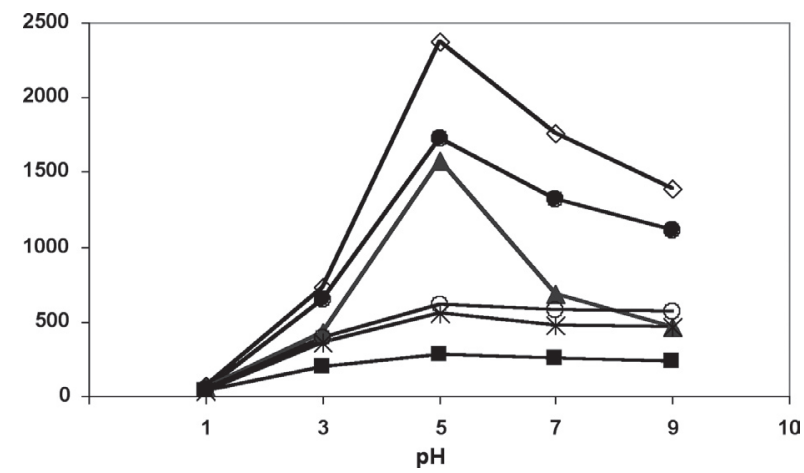

Figure 9. Absorption (Q) of poly (NNDMAAm-co-AMPS) 1:2 /

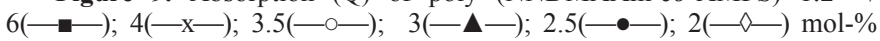
MBA in water at room temperature for $\mathrm{pH} 1,3,5,7$, and 9 . 
Effect of the temperature on absorbency

The effect of the temperature on the absorbency and water loss for poly(NNDMAAm-co-AMPS) for these feed mol ratios is shown in Figures 10 and 11 respectively. It demonstrates that the absorbency increases as increases the temperature until $30^{\circ} \mathrm{C}$ for the copolymer cross-linked with 2 mol- $\%$ of MBA. At higher temperatures the lost one of water is significant in comparison to copolymers with greater percentage of cross-linked, and the lost of water for all copolymers is complete at $60^{\circ} \mathrm{C}$.

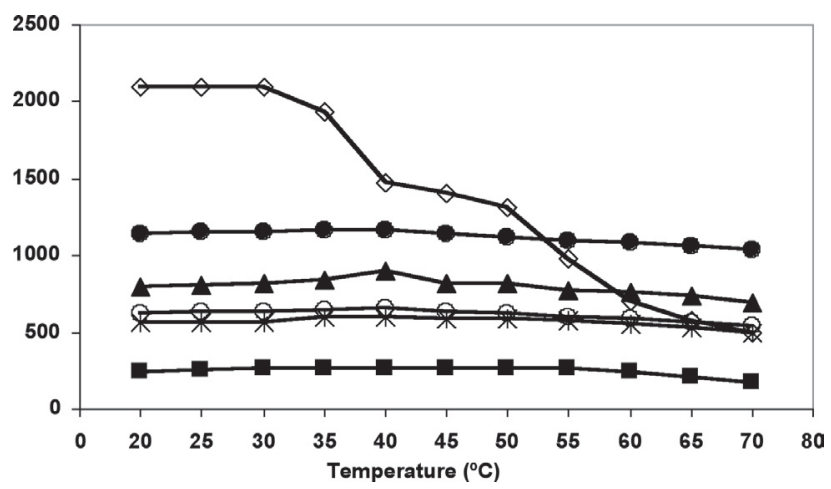

Figure 10. Absorption (Q) of poly(NNDMAAm-co-AMPS) 1:2

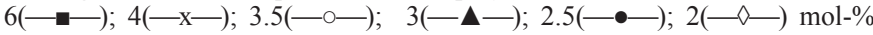
MBA at different temperatures for 24 hours.

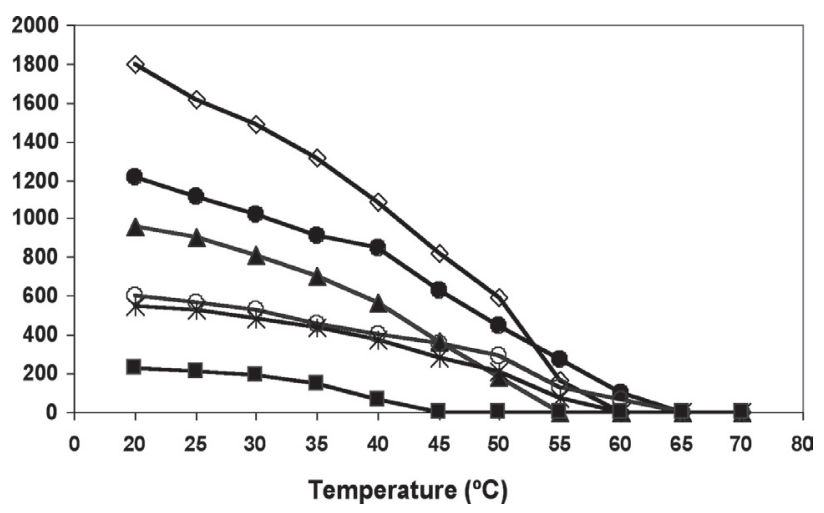

Figure 11. Water loss (Q) of poly(NNDMAAm-co-AMPS) $1: 2 /$

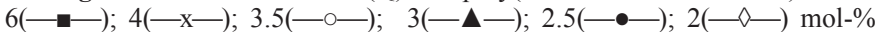
MBA at different temperatures for 24 hours.

\section{Effect of salt solutions on absorbency}

Figure 12 shows the effect of the solution's salt concentrations on the absorbency of the poly(NNDMAAm-co-AMPS). The results indicate that this polymer's absorbency depends in presence of the different $\mathrm{NaCl}$ concentrations on the copolymer composition. For the 1:2 copolymer in all its ratios of crosslinked a diminution in the swelling degree is observed up to $0.4 \mathrm{M}$.

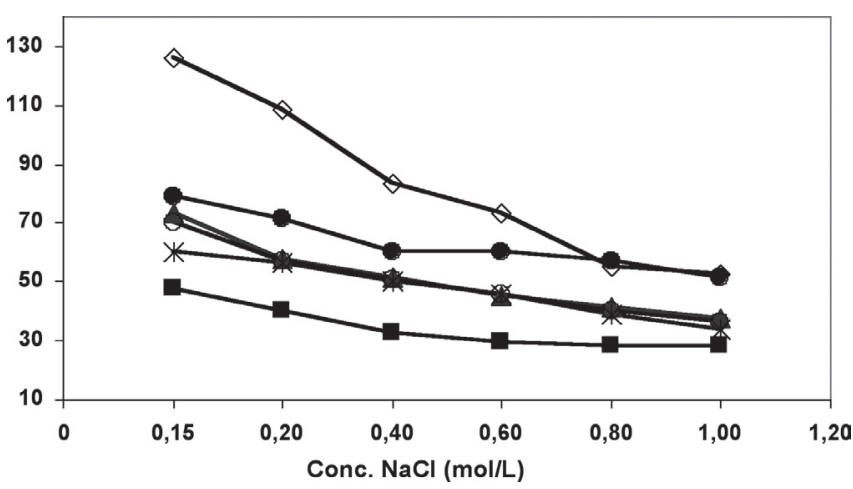

Figure 12. Absorption (Q) of poly(NNDMAAm-co-AMPS) $1: 2 /$

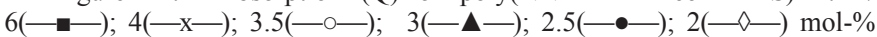
$\mathrm{MBA}$ at room temperature from different concentrations of $\mathrm{NaCl}$.

\section{CONCLUSIONS}

Hydrogels of NNDMAAm and AMPS were prepared in aqueous solution, and the copolymers showed lower critical solution temperature behavior (LCST). The phase transition temperatures of the aqueous solutions of these copolymer increased with increasing of hydrophilic comonomer AMPS contents. The LSCT was higher than poly(NIPAAm) ${ }^{11}$ at room temperature and thus, these polymers are suitable possible precursor polymers for the preparation of hydrogels for the development of temperature- and $\mathrm{pH}$-responsive switching devices through the sensitive gels with different properties.

The swelling properties showed that the poly(NNDMAAm-co-AMPS) has a higher absorbency in water with respect to ethanol and salt solutions. The absorbency of these polymers decreases as the solvent's polarity decreases.

The effects of $\mathrm{pH}$ on the absorbency were studied. It was found that the absorbency of poly(NNDMAAm-co-AMPS) 1:2 with 2 mol-\% MBA increases as the $\mathrm{pH}$ increases until 5 and a notable decrease in $\mathrm{pH}$ higher for the copolymer less cross-linked. The absorbency at pH 5 is still up to $2360 \mathrm{~g}$ water / g xerogel.

The influence of the salt solutions on the absorbency was investigated, and the results indicate that absorbency is a variable function of the rising salt concentration.

\section{ACKNOWLEDGEMENTS}

The authors thank the FONDECYT (Grant No 1050572) for financial support. BLR thanks to CIPA.

\section{REFERENCES}

1. S. Duran, D. Solpan, O. Güven, Nucl. Instrum. Methods Phys Res. Sect. B 151, 196 (1999).

2. HK. Ju, SY. Kim, SJ. Kim, YM. Lee, J. Appl. Polym. Sci. 83, 1128 (2002).

3. IY. Galacv, B. Mattiasson, TIBTECH 17, 335, (1999).

4. DM. Devine, CL. Higginbotham, Polymer 44, 7851 (2003).

5. K. Dušek, Adv. Polym. Sci. 109 \& 110, (1993)

6. D. De Rossi, K. Kajiwara, Y. Osada, A. Yamauchi, (eds) Polymer Gels. Fundamentals and Biomedical Applications. Plenum Press, New York (1991) .

7. R. Yoshida, K. Uchida, Y. Kaneko, K. Sakai, A. Kikuchi, Y. Sakurai, T. Okano, Nature 374, 240 (1995)

8. B.C .Shin, M.S. Jhon, H.B. Lee, S.H. Yuk, Eur. Polym. J. 34, 171, (1998).

9. D. Kuckling, A. Richter, KF. Arndt, Macromol. Mater Eng. 288, 144 , (2003).

10. B. Isik, Y. Gunay, J. Appl. Polym. Sci. 94,1619 (2004).

11. H.D. Schild, Progr. Polym. Sci. 17, 163, (1992).

12. M. Shibayama, T. Tanaka, Adv. Polym. Sci. 109, 1 (1993).

13. C. Wu, Polymer 39, 4609 (1998).

14. M. Hahn, E. Görnitz, H. Dautzenberg, Macromolecules 31, 5616 (1999).

15. D. Kuckling, H.J. Adler, L. Ling, W.D. Habicher, K.F. Arndt, Macromol. Chem. Phys. 201, 273 (2000). 
16. Y. Liu, J.L. Velada, M.B. Huglin, Polymer 40, 4299 (1999).

17. D. Kuckling, H.J. Adler, L. Ling, W.D. Habicher, K.F. Arndt, Polym. Bull. 44, 269 (2000).

18. C. Wu, S. Zhou, Macromolecules 28, 8381 (1995).
19. G. Chen, AS. Hoffman, Macromol. Chem. Phys. 196, 1251, (1995).

20. M. Shibayama, Y. Suetoh, S. Nomura, Macromolecules 29, 6966 (1996).

21. F. Tüdös, T. Kelen, B. Turcsanyi, J. Polym. Sci: Polym. Chem. Ed. 19, 1119, (1981). 